\title{
Lexis
}

Journal in English Lexicology

Book reviews | 2007

\section{Werner HÜLLEN, English Dictionaries, 800-1700. The}

Topical Tradition

Oxford University Press Ltd, 2006, 525 pages

\section{Henri Béjoint}

\section{OpenEdition}

\section{Journals}

Édition électronique

URL : http://journals.openedition.org/lexis/1343

DOI : $10.4000 /$ lexis. 1343

ISSN : 1951-6215

Éditeur

Université Jean Moulin - Lyon 3

Référence électronique

Henri Béjoint, «Werner HüLlen, English Dictionaries, 800-1700. The Topical Tradition », Lexis [En ligne], Recensions, mis en ligne le 21 mars 2007, consulté le 24 septembre 2020. URL : http:// journals.openedition.org/lexis/1343; DOI : https://doi.org/10.4000/lexis.1343

Ce document a été généré automatiquement le 24 septembre 2020.

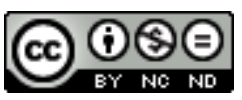

Lexis is licensed under a Creative Commons Attribution-NonCommercial-NoDerivatives 4.0 International License. 


\title{
Werner HÜLLEN, English Dictionaries, 800-1700. The Topical Tradition
}

Oxford University Press Ltd, 2006, 525 pages

\author{
Henri Béjoint
}

\section{RÉFÉRENCE}

Werner Hüllen

English Dictionaries, 800-1700. The Topical Tradition. Oxford University Press Ltd, 2006.

ISBN : 0199291047 , Prix : $230 €, 525$ pages

1 Werner Hüllen a beaucoup écrit, surtout sur la lexicographie onomasiologique. Il est en particulier l'auteur de la grande étude sur le Thesaurus de Roget (Hüllen 2004). Le présent ouvrage est une nouvelle édition, en paperback, de son livre de 1999 sous le même titre, signe révélateur de son succès. Il compte 525 pages, dont quarante pages d'annexes et une impressionnante bibliographie [1] .

2 La lexicographie de langue anglaise est très étudiée. Pour les périodes les plus anciennes, on peut utiliser, entre autres, Starnes et Noyes (1946), Stein (1985), McArthur (1986) ou Green (1997). Dans ce nouvel ouvrage, plus spécialisé, Werner Hüllen se propose d'écrire les premières pages, de 800 à 1700, d'une histoire de la tradition onomasiologique (qu'il appelle topical), dont il dit (35) qu'elle a été injustement négligée par les métalexicographes. La tâche est rude : on dit volontiers que ces dictionnaires sont tellement compliqués que seuls les gens très instruits peuvent s'en servir, ceux-là même qui n'en ont pas besoin (13). Il ne s'agit pas de raviver la vieille querelle sur l'onomasiologie qui a fait rage au début du XXe siècle, qui de toute façon n'a plus lieu d'être grâce aux progrès de la linguistique (21) (et aussi grâce à ceux de l'informatique, dont Werner Hüllen ne parle pas), mais d'expliquer comment et pourquoi la lexicographie onomasiologique est un courant ancien, intéressant et important. 
3 Le livre est divisé en quatre grandes parties, divisées elles-mêmes en chapitres. La première partie, Opening the topic, commence par la définition de quelques termes fondamentaux. D'abord, la lexicographie, que Werner Hüllen considère, en tant que sous-discipline de la linguistique, comme une théorie qui gouverne la production de dictionnaires (5). La lexicographie exploite la linguistique, la recherche sur l'utilisation des dictionnaires et l'histoire de la pratique lexicographique. Werner Hüllen revient ensuite sur la distinction entre le savoir sur le monde (world knowledge) et le savoir sur les mots (word knowledge) : le premier est omniprésent dans tous les dictionnaires, car c'est lui qui permet de comprendre les définitions (8 sq.). Puis Werner Hüllen présente l'ordre alphabétique, adopté par la quasi-totalité des dictionnaires au fil de leur histoire, sans doute parce que les premiers dictionnaires européens étaient bilingues. Il convient aux besoins du récepteur du message, et singulièrement du traducteur. Pour Werner Hüllen, il va de l'inconnu, à gauche de l'équation, au connu, à droite (11 sq.), alors que les ouvrages onomasiologiques [2] procèdent à l'inverse, du connu vers l'inconnu (13). L'opposition mériterait discussion. En tout cas, les ouvrages onomasiologiques supposent une organisation du savoir très difficile à mettre au point, à laquelle les lexicographes ont accordé une importance peut-être excessive (15) au regard de leurs objectifs pratiques. Les dictionnaires onomasiologiques sont des textes, dit ensuite Werner Hüllen (22) : ils sont composés d'un niveau " micro », l'article et d'un niveau "meso", la section, tous deux insérés dans le niveau "macro », le dictionnaire tout entier (22). C'est au niveau «meso » qu'intervient l'organisation du savoir fondée sur une conception du monde (24).

4 Werner Hüllen passe ensuite à des considérations méthodologiques. Il définit avec beaucoup de soin ce qu'est une tradition (28) et ce qu'est une méthode de recherche scientifique. Il aura soin, tout au long du livre, de donner des détails sur les méthodes qu'il utilise $(95,192,398$, etc.), souci de rigueur et de précision qui l'honore mais qui alourdit sensiblement son texte. La fin de cette première partie est consacrée à la peinture du décor de son étude : les listes onomasiologiques existent depuis la nuit des temps dans les civilisations égyptiennes, chinoises, sanscrites, gréco-coptes et arabosyriaques, et on ne peut qu'être frappé par la similitude des ouvrages, même dans les cas où il ne peut pas être question d'influences directes ou indirectes (35).

5 La deuxième partie, The English Tradition of Onomasiology (41) est la plus longue. Elle est consacrée à l'examen de dictionnaires onomasiologiques anglais de la période, dans l'ordre chronologique de leur parution. Différents types peuvent être dégagés: le glossaire text-dependent (56), les glossae collectae, etc., qui s'ajoutent aux listes onomasiologiques proprement dites et aux glossaires alphabétiques. Les recueils onomasiologiques sont historiquement liés à l'enseignement des langues, d'abord le latin puis les langues vernaculaires (à partir du dernier tiers du XVe siècle, 105), singulièrement le français après 1066 (87). Werner Hüllen examine successivement les Hermeneumata, listes de mots destinées à être apprises par cœur, où le classement sert surtout à faciliter la mémorisation (50), le Glossary d'Aelfric (qui doit beaucoup à Isidore de Séville) et le Mayer Nominale. Tous ces exemples précoces traitent en gros les mêmes thèmes, que Werner Hüllen détaille soigneusement, et sont des témoins précieux de la vie de leur époque.

6 Werner Hüllen aborde ensuite le domaine des colloquies (78), recueils de vocabulaire présentés comme des échanges de maître à élève, qui contiennent des listes de mots apparentés sémantiquement. Parmi eux, celui d'Érasme et le fameux Dictionarius de 
John of Garland, publié à Paris en 1220 (85), qui est le premier à avoir utilisé ce titre. Puis viennent les conversation books, dont le principal est celui de Caxton, Dialogues in French and English, publié en 1483, dont Werner Hüllen décrit longuement le contenu et les objectifs (93-104, puis encore 133-139). On arrive ensuite aux XVIe et XVIIe siècles (104), avec leurs dialogues, dont celui de Berlaimont, publié en 1530, qui compta jusqu'à huit langues, y compris le latin, qui fut utilisé dans toute l'Europe jusqu'au début du XVIIIe siècle (30 000 copies!, 112) et dont Werner Hüllen décrit en grand détail les différentes éditions (106-118), et celui de Guy Miège, bien connu des lexicographes. Tous contiennent des indications intéressantes, dit Werner Hüllen, sur la société de leur temps, et des allusions à des éléments réels, villes, personnes, événements, monnaies, etc., mais il ne faut pas les prendre pour des miroirs (132) : nombreux étaient les termes désignant des choses imaginées, racontées, reprises d'auteurs anciens, des animaux, des plantes, des maladies, etc. que personne n'avait jamais vus mais dont les noms se transmettaient par tradition de livre en livre. Les descriptions de l'époque n'étaient pas plus ressemblantes que ne l'étaient les portraits.

7 Le chapitre suivant aborde les listes onomasiologiques spécialisées (140). Werner Hüllen note que de nombreux recueils des termes d'un domaine technique ou scientifique particulier paraissent avant Bacon (1561-1626), à qui on ne peut donc pas attribuer la paternité du savoir scientifique appliqué. Ils ont été un peu plus étudiés que les recueils examinés dans le chapitre précédent, en particulier en tant que sources pour les dictionnaires monolingues. On voit poindre en eux, déjà, ce qui sera la marque $\mathrm{du}$ siècle des lumières et qu'on trouvera chez les encyclopédistes, que le bonheur est dans le savoir (155). Werner Hüllen décrit des manuels de husbandry (142), de rhétorique (149), de géométrie (154), de navigation (John Smith, 159), etc. À chaque fois, Werner Hüllen donne des détails sur le contenu, l'organisation, le ton, etc. On en arrive ensuite à John Withals (168), prédécesseur trop mal connu de Comenius, dont le dictionnaire, publié en 1553, fut l'objet de nombreuses rééditions de plus en plus volumineuses (jusqu'à 18400 mots d'après Werner Hüllen, 172) qui restèrent extrêmement populaires pendant plus de quatre-vingts ans (169). Werner Hüllen lui consacre plus de 30 pages. C'est une liste d'expressions anglaises avec leurs équivalents en latin, destinée à l'enseignement du latin aux jeunes Anglais. Les mots y sont arrangés par thèmes, dans une organisation où l'église et la religion n'occupent plus la place pré-éminente, contrairement aux ouvrages des siècles précédents (180).

L'ouvrage examiné ensuite, le Lexicon Tetraglotton de James Howell (1660), est moins connu. Werner Hüllen lui consacre pourtant 40 pages. Il s'agit, comme son titre l'indique, d'un dictionnaire en quatre langues, composé, comme son titre ne l'indique pas, d'une partie alphabétique et d'une partie onomasiologique (204), cette dernière étant adaptée d'un ouvrage italien (218), ce qui souligne une fois de plus à quel point les lexicographes de l'époque étaient ouverts aux influences par-delà les frontières. Werner Hüllen passe ensuite (244) à l'ouvrage inclassable de John Wilkins (1668), Essay towards a Real Character and a Philosophical Language, qui est sans doute le plus important de toute l'histoire de la lexicographie onomasiologique anglaise avant Roget, dont il sera d'ailleurs l'inspirateur principal. L'ouvrage se rattache au mouvement général en Europe pour la mise au point d'une langue universelle, auquel appartiennent Bacon, Comenius, Descartes ou Leibniz. Werner Hüllen lui consacre 57 pages, dont cinq de reproductions des pages de Wilkins (on aurait aimé en voir aussi pour les autres ouvrages examinés, mais il est vrai que les éditeurs reculent souvent devant la dépense). Wilkins part de l'idée que tous les hommes sont à peu près d'accord sur ce 
qu'il est nécessaire de nommer, et propose donc une liste de lexèmes qui se veulent « alinguistiques ", universaux, qui sont autant de slots qui ne demandent qu'à être remplis par des mots réels d'une langue donnée (264). Son ouvrage est fait de plusieurs parties qui se complètent et se répondent, et chaque mot y apparait plusieurs fois. Il est original et copieux, d'une extraordinaire complexité, d'une importance dont l'auteur lui-même ne s'est probablement pas rendu compte, et on peut le voir comme une préfiguration du mouvement encyclopédique (286), au même titre que le Pseudodoxia Epidemica de Thomas Browne (dont Werner Hüllen ne parle pas), paru en 1646, même si les ouvrages sont très différents [3]. La classification des plantes, en particulier (262), annonce celle que proposera Linné un siècle plus tard. Remarquable tentative, même si elle se révéla finalement mal conçue, selon Werner Hüllen (252), parce qu'elle oscille entre le désir de mise au point d'une langue universelle et celui de représentation du lexique anglais.

La troisième partie, The European Scene (1400-1700) (303), élargit l'examen à l'Europe. Le chapitre 9 revient curieusement en arrière dans le temps. Il examine la production de livres depuis l'invention de l'imprimerie jusqu'en 1600: dictionnaires onomasiologiques destinés à enseigner le latin (309), avec ou sans le grec et l'hébreu, dictionnaires multilingues de toutes sortes, nomenclators utilisés dans le trivium (321), manuels de langues vernaculaires (326), successeurs et imitateurs du fameux Introito $e$ porta (336). Werner Hüllen peint un vaste portrait de la production et du commerce des livres utilisés pour l'enseignement et l'apprentissage des langues dans l'Europe du XVIe siècle, marquée par la prédominance du latin (318), la «montée en puissance » des langues vernaculaires, et le plurilinguisme. Les chiffres de ventes de ces ouvrages sont parfois étonnants (315). Le chapitre 10 (361) est consacré tout entier (70 pages) à Comenius (1592-1670). Werner Hüllen le replace d'abord dans le contexte de la lexicographie du tchèque, même s'il souligne, à juste titre, que la culture de l'époque, et à plus forte raison ses figures les plus marquantes, ne peuvent être comprises qu'au niveau de l'ensemble du continent, et non d'une seule communauté linguistique. Les deux œuvres principales de Comenius, Janua Linguarum Reserata (1631) et Orbis Sensualium Pictus (1658), sont destinées à l'enseignement du latin (et d'autres langues dans des éditions ultérieures), mais elles sont surtout remarquables par la vision du monde qu'elles ambitionnent de transmettre. Comenius distingue trois niveaux: les choses, l'idée que l'homme se fait des choses, et les mots qu'il utilise pour désigner ces idées (372 sq.). Janua Linguarum Reserata, dictionnaire de phrases (387), connut 101 rééditions sous diverses formes, avec toutes les langues européennes (et même en arabe, turc, persan et mongol 385) pendant tout le siècle. Il fut même utilisé dans la toute nouvelle université d'Harvard aux Amériques! En tout, Werner Hüllen calcule que 30.000 copies environ ont circulé (385). Orbis Sensualium Pictus (392) est une collection d'images plus ou moins complexes, dont les éléments sont numérotés (Werner Hüllen en reproduit 9 exemplaires, hélas pas toujours à la bonne page) destinée à de jeunes élèves. Les illustrations sont "glosées" par des phrases qui utilisent les mêmes numéros, ce qui en fait prototype du dictionnaire onomasiologique (393) et l'ancêtre de la grande Encyclopédie, et évidemment des Duden modernes (dont Werner Hüllen ne parle pas). Le livre connut 245 éditions, dont 215 avec le latin (419), et plusieurs pour l'Angleterre.

10 La quatrième partie, Reflections on the topic (431), est une sorte d'envoi faisant écho à la première. Werner Hüllen retrace (435) la tradition encyclopédique européenne, Pline l'ancien (23-79), Isidore de Séville (c.560-636), Bede le vénérable (676-735), puis, après 
plusieurs siècles d'activité plus réduite, Barthélémy l'Anglois (fin du XIIe, début du XIIIe siècles), dont on ne sait pas grand-chose, Caxton (1481), etc. D'une certaine manière, tous les ouvrages onomasiologiques appartiennent à cette tradition (439), comme ils appartiennent aussi à la tradition terminologique de la nomenclature, qui suppose la pré-existence du réel et postule le "mot-étiquette». Le dictionnaire onomasiologique s'épuise vers 1700 , dit Werner Hüllen, avec l'avènement de la philosophie de John Locke (Essay on Human Understanding, 1689), pour qui les mots ne peuvent pas être les étiquettes des choses mais sont les représentants des idées formées par les hommes. Il laisse la place au dictionnaire alphabétique (444), et ne réapparaîtra qu'au milieu du XIXe siècle avec le Thesaurus de Roget.

11 Werner Hüllen atteint-il son objectif ? Globalement oui, même si dans un ouvrage d'une telle dimension et d'une telle ambition on trouvera toujours des points avec lesquels on ne sera pas d'accord ou qu'on aurait aimé voir développés (l'informatique, la question des variétés de langue, etc.). La tradition onomasiologique est née d'un rêve des hommes de faire coïncider les mots avec les choses, et elle a produit des ouvrages souvent intéressants, parfois fascinants ou pathétiques dans leurs tentatives, mais éloignés du rêve. L'avènement de l'informatique, dont Werner Hüllen ne parle pas, pourrait bien lui donner un nouveau souffle. On voit bien comment une base de données comme WordNet, par exemple, pourrait être retournée pour pouvoir être utilisée en partant du concept pour aller vers le signe.

Le livre de Werner Hüllen est celui d'un spécialiste de l'histoire des idées, marqué par le souci de l'historien pour la précision et le recours aux sources primaires et par celui du lexicographe pour l'étiquetage des idées et des choses. On imagine aisément Werner Hüllen tenant précautionneusement entre ses mains les ouvrages qu'il décrit, et on suppute les heures passées au British Museum et dans d'autres vénérables bibliothèques [4] .

Le style de Werner Hüllen est parfois pesant et répétitif, la lecture pas toujours facile et les 500 pages d'une typographie serrée pourront faire hésiter certains. Mais ils auraient tort, car le livre a de quoi récompenser leurs efforts. Sérieux, documenté, plein à ras bords de choses que l'on ne trouvera que difficilement, voire pas du tout ailleurs, il pourra, avec son index (hélas pas assez détaillé), servir d'ouvrage de référence. Werner Hüllen mérite des remerciements, et des éloges pour la somme qu'il nous offre sur un sujet trop négligé.

[1] Avec hélas quelques fautes typographiques. Eh oui, même dans un volume publié par Oxford University Press...

[2] Werner Hüllen mentionne d'autres adjectifs utilisés pour désigner ces ouvrages : en anglais, outre topical, il y a thematic, conceptual, ideographical (13), systematic (141), en français analogique, idéologique (16).

[3] L'ouvrage de John Harris, Lexicon Technicum, paraîtra en 1704, et la Cyclopaedia d'Ephraim Chambers en 1728.

[4] Presque tous les ouvrages qu'il mentionne sont désormais disponibles sur le site Early English Books Online. Il ne semble pas que Werner Hüllen y ait eu accès. 


\section{BIBLIOGRAPHIE}

GREEN, J., Chasing the Sun :Dictionary Makers and the Dictionaries They Made, New York, Henry Holt, 1996.

HÜLLEN, W.), A History of Roget's Thesaurus, Oxford, Oxford University Press, 2004.

MCARTHUR, T., Worlds of Reference, Cambridge, Cambridge University Press, 1986.

STARNES, D. T. \& NOYES, G. E., The English Dictionary from Cawdrey to Johnson (1604-1755), Chapel Hill, University of North Carolina Press, 1946. Deuxième édition : avec une Introduction et une nouvelle bibliographie de G. Stein, Amsterdam / Philadelphia, Benjamins, 1991.

STEIN, G., The English Dictionary before Cawdrey, Tübingen, Niemeyer, 1985.

\section{AUTEURS}

\section{HENRI BÉJOINT}

Henri Béjoint, Département de Langues Étrangères Appliquées, Université Lumière-Lyon 2, France.

Henri Béjoint enseigne la traduction anglais - français, la terminologie, la lexicologie et la lexicographie depuis 1973. Il est l'auteur de nombreux articles et ouvrages, notamment Modern Lexicography: An Introduction, publié par Oxford University Press (2001). Il dirige le Centre de Recherche en Terminologie et Traduction (CRTT). 